Research Article

\title{
Cytoreductive Surgery plus Hyperthermic Intraperitoneal Chemotherapy Improves Survival with Acceptable Safety for Advanced Ovarian Cancer: A Clinical Study of 100 Patients
}

\author{
Jue Zhang, ${ }^{1}$ Xin-bao Li, ${ }^{1}$ Zhong-he Ji, ${ }^{1}$ Ru Ma, ${ }^{1}$ Wen-pei Bai, ${ }^{2}$ and Yan Li $i^{1}$ \\ ${ }^{1}$ Department of Peritoneal Cancer Surgery, Beijing Shijitan Hospital, Capital Medical University, China \\ ${ }^{2}$ Department of Gynecology, Beijing Shijitan Hospital, Capital Medical University, China \\ Correspondence should be addressed to Yan Li; liyansd2@163.com
}

Received 24 January 2021; Revised 28 March 2021; Accepted 17 May 2021; Published 23 June 2021

Academic Editor: Qiang Liu

Copyright (c) 2021 Jue Zhang et al. This is an open access article distributed under the Creative Commons Attribution License, which permits unrestricted use, distribution, and reproduction in any medium, provided the original work is properly cited.

\begin{abstract}
Background. The mainstay of treatment for advanced ovarian cancer is debulking surgery followed by chemotherapy that includes carboplatin and paclitaxel, but the prognosis is poor. This study is aimed at evaluating the efficacy and safety of cytoreductive surgery plus hyperthermic intraperitoneal chemotherapy (CRS+HIPEC) as first-line surgical treatment in patients with advanced ovarian cancer (AOC). Methods. FIGO stage III/IV AOC patients underwent CRS+HIPEC as first-line surgical treatment at our center from December 2007 to January 2020. The primary endpoint was survival, and the secondary endpoint was safety. Results. Among 100 patients, the median Karnofsky performance status (KPS) score was 80 (50-100), median peritoneal cancer index (PCI) was 19 (1-39), median completeness of cytoreduction (CC) score was 1 (0-3), number of organ regions removed was 4 (3-9), number of peritoneal regions removed was 4 (1-9), and number of anastomoses was 1 (0-4). The median follow-up was 36.8 months; 75 (75.0\%) patients were still alive, and 25 (25.0\%) had died. The median overall survival (mOS) was 87.6 (95\% CI: 72.1-103.0) months, and the 1-, 2-, 3-, 4-, and 5-year survival rates were 94.1\%, 77.2\%, 68.2\%, 64.2\%, and $64.2 \%$, respectively. Univariate analysis showed that better $\mathrm{mOS}$ correlated with an age $\leq$, KPS $\geq 80$, ascites $\leq 1000 \mathrm{ml}$, $\mathrm{PCI}<19$, and CC score 0-1. Multivariate Cox analysis showed that CC was an independent factor for OS; patients who underwent CRS with a CC score 0-1 had a mPFS of 67.8 (95\% CI: 48.3-87.4) months. The perioperative serious adverse event and morbidity rates were $4.0 \%$ and $2.0 \%$, respectively. Conclusions. CRS+HIPEC improves survival for AOC patients with acceptable safety at experienced high-volume centers. Stringent patient selection and complete CRS are key factors for better survival.
\end{abstract}

\section{Introduction}

The majority of patients with ovarian cancer (OC) are diagnosed with advanced disease that has spread beyond the ovaries to cause peritoneal metastasis (PM), and this advanced stage accounts for the highest mortality of all gynecologic cancers [1]. The fatal manifestation of cancer dissemination on the omentum, peritoneum, and mesentery leads to refractory ascites, progressive intestinal obstruction, and intractable abdominal pain associated with early death and a miserable quality of life for such patients $[2,3]$. Even after the standard treatment of optimal debulking surgery followed by intravenous platinum/taxane-based chemother- apy for advanced ovarian cancer (AOC) $[4,5], 75 \%$ of patients still develop recurrence and present with PM [6], which is the most difficult obstacle to improving AOC treatment.

Over the past three decades, aggressive cytoreductive surgery (CRS) plus hyperthermic intraperitoneal chemotherapy (HIPEC) has been developed as a comprehensive treatment package integrating multivisceral resections to remove macroscopic residual tumors, and HIPEC has been used to treat residual cancer cells after CRS $[7,8]$.

Bakrin et al. [9] report a multicenter retrospective cohort study included 566 patients, 92 patients with primary EOCPM, and 474 patients with recurrent EOCPM, which 
Peritoneal cancer Index

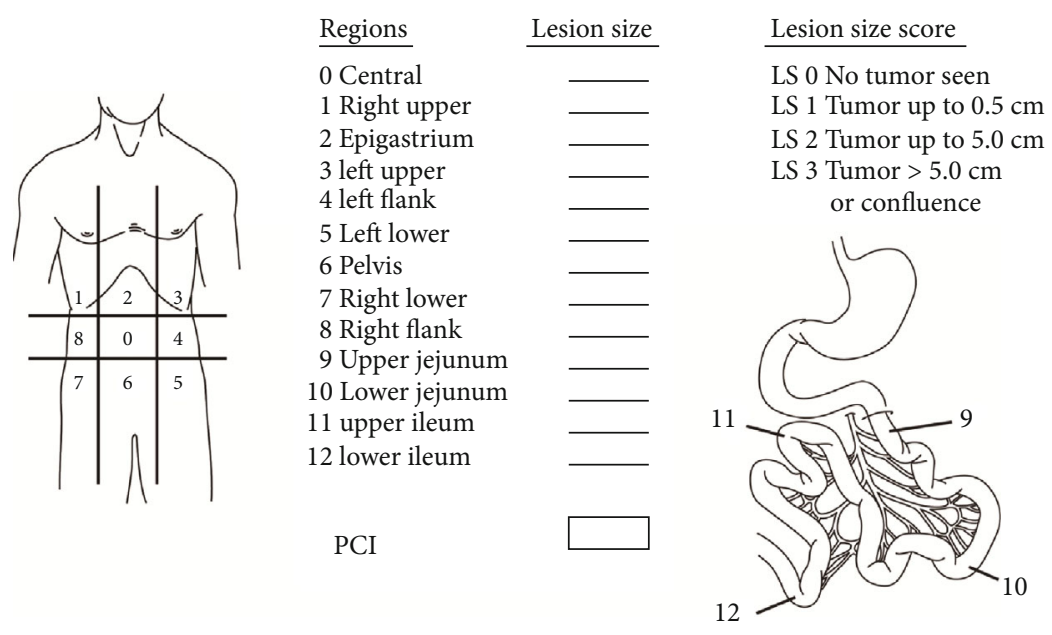

(a)

Completeness of cytoreduction after surgery (CC score)

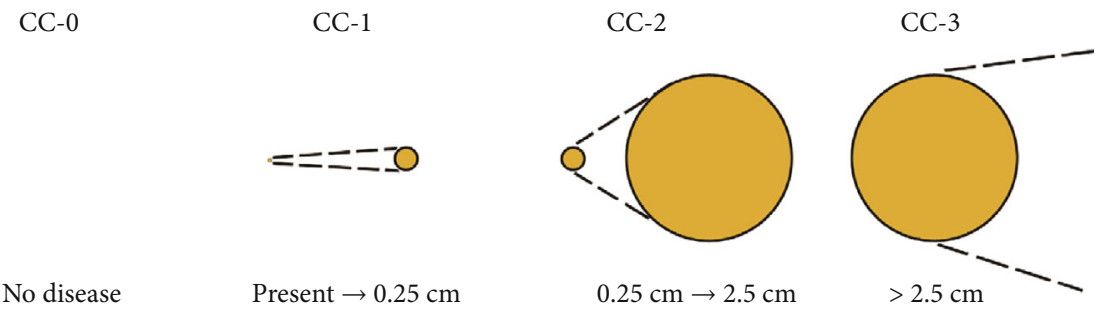

(b)

FIgURE 1: Graphical representation of PCI and CC score according to the Sugarbaker [8].

the CRS+HIPEC as first-line treatment. The median overall survivals (mOSs) were 35.4 months and 45.7 months for advanced and recurrent EOC, respectively. van Driel et al. [10] report a first RCT to compare the efficacy and safety of CRS+HIPEC and CRS groups, and the results showed the survival gain in the CRS+HIPEC group. Although CRS+HIPEC is considered one of several acceptable options for patients with stage III/IV OC by the National Comprehensive Cancer Network (NCCN) guidelines [11], there is currently limited evidence from clinical studies to definitively determine the efficacy and safety of standard operation programs and patient selection criteria.

A retrospective analysis was conducted in this study to assess the efficacy and safety of CRS+HIPEC as a front-line surgical regimen in 100 patients with AOC.

\section{Methods and Materials}

2.1. Patient Selection. From December 1, 2007, to January 1, 2020, 100 patients diagnosed with FIGO stage III/IV OC underwent CRS+HIPEC as a first-line surgery strategy at our center. Informed consent was obtained from all patients, and the study was approved by the institutional review board and the ethics committee.
The main inclusion criteria were as follows: (1) no history of PM-related surgery; (2) peripheral white blood cell count $\geq 3,500 / \mathrm{mm}^{3}$ and platelet count $\geq 8,000 / \mathrm{mm}^{3}$; (3) acceptable liver function, with bilirubin $\leq 2 \times$ the upper limit of normal (ULN) and aspartic aminotransferase (AST) and alanine aminotransferase $(\mathrm{ALT}) \leq 2 \times \mathrm{ULN}$; (4) acceptable renal function, with serum creatinine $(\mathrm{Scr}) \leq 1.5 \mathrm{mg} / \mathrm{dl}$; (5) adequate cardiovascular and pulmonary function and function of other major organs that could tolerate major operation; and (6) Karnofsky performance status (KPS) $\geq 50$.

The major exclusion criteria were as follows: (1) history of PM-related surgery; (2) any lung, liver, or prominent retroperitoneal lymph node metastases found during preoperative assessment; (3) imaging examination showing obvious contractures of the mesentery; (4) serum bilirubin level $>3$ $\times \mathrm{ULN}$ and ALT/AST $\geq 2 \times \mathrm{ULN}$; (5) liver enzymes $>3 \times$ ULN; (6) Scr > $1.5 \mathrm{mg} / \mathrm{dl}$; (7) KPS score $<50$; and (8) absence of peritoneal metastasis.

2.2. Preoperative Evaluation. Patients were evaluated according to the Chinese expert consensus [12] of CRS+HIPEC, which included the following: (1) physical examination: serum tumor marker levels including carbohydrate antigen 125 (CA 125, normal range: 0-35 U/mL), carbohydrate antigen 199 (CA 19-9, normal range: 0- 
TABle 1: Demographic and clinical characteristics of 100 AOC patients.

\begin{tabular}{|c|c|}
\hline Items & Value \\
\hline \multicolumn{2}{|l|}{ Clinical characteristics } \\
\hline Age (median, range) (y) & $58.5(28-87)$ \\
\hline KPS score (median, range) & $80(50-100)$ \\
\hline \multicolumn{2}{|l|}{ History of chemotherapy $(n, \%)$} \\
\hline Yes & $52(52.0)$ \\
\hline No & $48(48.0)$ \\
\hline \multicolumn{2}{|l|}{ Histopathology $(n, \%)$} \\
\hline Serous carcinoma & $91(91.0)$ \\
\hline Other types & $9(9.0)$ \\
\hline Cycles of SC before surgery (median, range) & $3(0-45)$ \\
\hline $\begin{array}{l}\text { Cycles of IPC before surgery } \\
\text { (median, range) }\end{array}$ & $1(0-9)$ \\
\hline Cycles of SC after surgery (median, range) & $4(0-26)$ \\
\hline Cycles of IPC after surgery (median, range) & $6(0-8)$ \\
\hline SC before surgery $(n, \%)$ & $48(48)$ \\
\hline IPC before surgery $(n, \%)$ & $16(16)$ \\
\hline SC after surgery $(n, \%)$ & $76(76)$ \\
\hline IPC after surgery $(n, \%)$ & $46(46)$ \\
\hline \multicolumn{2}{|l|}{ CRS+HIPEC relevant parameters } \\
\hline \multicolumn{2}{|l|}{ Organ regions resected $(n, \%)$} \\
\hline $1-3$ resections & $39(39.0)$ \\
\hline$>4$ resections & $61(61.0)$ \\
\hline \multicolumn{2}{|l|}{ Peritoneal regions resected $(n, \%)$} \\
\hline 0-3 resections & $37(37.0)$ \\
\hline 4-6 resections & $40(40.0)$ \\
\hline$>6$ resections & $23(23.0)$ \\
\hline \multicolumn{2}{|l|}{ Number of anastomosis $(n, \%)$} \\
\hline $0-1$ & $34(34.0)$ \\
\hline$\geq 1$ & $66(66.0)$ \\
\hline \multicolumn{2}{|l|}{ PCI score $(n, \%)$} \\
\hline$\leq 19$ & $53(53.0)$ \\
\hline$>19$ & $47(47.0)$ \\
\hline \multicolumn{2}{|l|}{ CC score $(n, \%)$} \\
\hline $0-1$ & $79(79.0)$ \\
\hline $2-3$ & $21(21.0)$ \\
\hline \multicolumn{2}{|l|}{ Lymph node dissection $(n, \%)$} \\
\hline Pelvic lymph node & $100(100)$ \\
\hline Abdominal aortic lymph node & $68(68)$ \\
\hline Iliac lymph nodes & $100(100)$ \\
\hline \multicolumn{2}{|c|}{ Fluid output volume at surgery (median, range) } \\
\hline Blood loss (ml) & $550(0-3,000)$ \\
\hline Urine output (ml) & $1,500(300-4,500)$ \\
\hline Ascites (ml) & $270(0-8,000)$ \\
\hline$\leq 1000(n, \%)$ & $68(68.0)$ \\
\hline$>1000(n, \%)$ & $32(32.0)$ \\
\hline
\end{tabular}

TABle 1: Continued.

\begin{tabular}{|c|c|}
\hline Items & Value \\
\hline \multicolumn{2}{|c|}{ Fluid intake volume at surgery (median, range) } \\
\hline Plasma (ml) & $600(0-4,000)$ \\
\hline $\mathrm{RBC}(\mathrm{U})^{\mathrm{a}}$ & $2(0-12.0)$ \\
\hline Other fluids $(\mathrm{ml})^{\mathrm{b}}$ & $\begin{array}{c}6,735(100- \\
13,950)\end{array}$ \\
\hline $\begin{array}{l}\text { CRS+HIPEC duration } \\
\text { (median, range) }(\mathrm{min})\end{array}$ & $600(80-910)$ \\
\hline Stay in hospital (median, range) (d) & $27(0-120)$ \\
\hline \multicolumn{2}{|c|}{${ }^{\mathrm{a}} 1 \mathrm{U}=200 \mathrm{ml} .{ }^{\mathrm{b}}$ Including crystalloid, colloidal fluid injection volume. } \\
\hline \multicolumn{2}{|c|}{$\begin{array}{l}37 \mathrm{U} / \mathrm{mL} \text { ), and carcinoembryonic antigen (CEA, normal } \\
\text { range: } 0-5 \mathrm{ng} / \mathrm{mL} \text { ); ( } 2 \text { ) imaging examination: abdominopel- } \\
\text { vic multidetector computed tomography (CT) plus multi- } \\
\text { planar reconstruction to evaluate gastrointestinal motility, } \\
\text { intestinal obstruction, and mesenteric contracture; and } \\
\text { (3) cytology: cytological examination of ascites or exfoliat- } \\
\text { ing cells from peritoneal washing fluid. }\end{array}$} \\
\hline
\end{tabular}

2.3. CRS+HIPEC Procedures. All CRS+HIPEC procedures were conducted by a designated team focusing on PM treatment. Briefly, abdominal exploration was performed through a midline xiphoid-to-pubis incision after administering general anesthesia, and the peritoneal cancer index (PCI) was evaluated and recorded. Then, maximal CRS was performed, including curative or palliative resection of the primary tumor with acceptable margins an any involved adjacent structures, lymphadenectomy, and peritoneal resection, and then, the completeness of cytoreduction (CC) score was calculated.

Open HIPEC was implemented with each drug dissolved in $3 \mathrm{~L}$ of heated saline at $43 \pm 0.5^{\circ} \mathrm{C}$, and the duration of HIPEC was $60 \mathrm{~min}$ with a flow rate of $400 \mathrm{~mL} / \mathrm{min}$. The HIPEC regimens included docetaxel(DTX) $120 \mathrm{mg}+$ cisplatin (DDP) $120 \mathrm{mg}$, DTX $120 \mathrm{mg}+$ mitomycin C (MMC ) $30 \mathrm{mg}$ for patients with high-risk factors for renal dysfunction, and DTX $120 \mathrm{mg}$ only for patients with a single kidney and/or impaired renal function confirmed by laboratory tests.

After the operation, the patients were transferred to the intensive care unit for recovery and then to the ward when they stabilized.

2.4. Postoperative Chemotherapy. Adjuvant chemotherapy was delivered within 6 to 8 weeks after CRS+HIPEC, including platinum/taxane-based systematic chemotherapy (SC) and perioperative intraperitoneal chemotherapy (IPC) through the IPC port once every 4 to 6 weeks. DDP $100 \mathrm{mg} / \mathrm{m}^{2}$ and paclitaxel/DTX $100 \mathrm{mg} / \mathrm{m}^{2}$ were administered.

2.5. Follow-Up. All patients were regularly followed up once every 3 months for the first 2 years, every 6 months for years 3 to 5 , and every year thereafter to obtain detailed information on disease status. The most recent follow-up was performed on January 1, 2020, and no patients have been lost. 


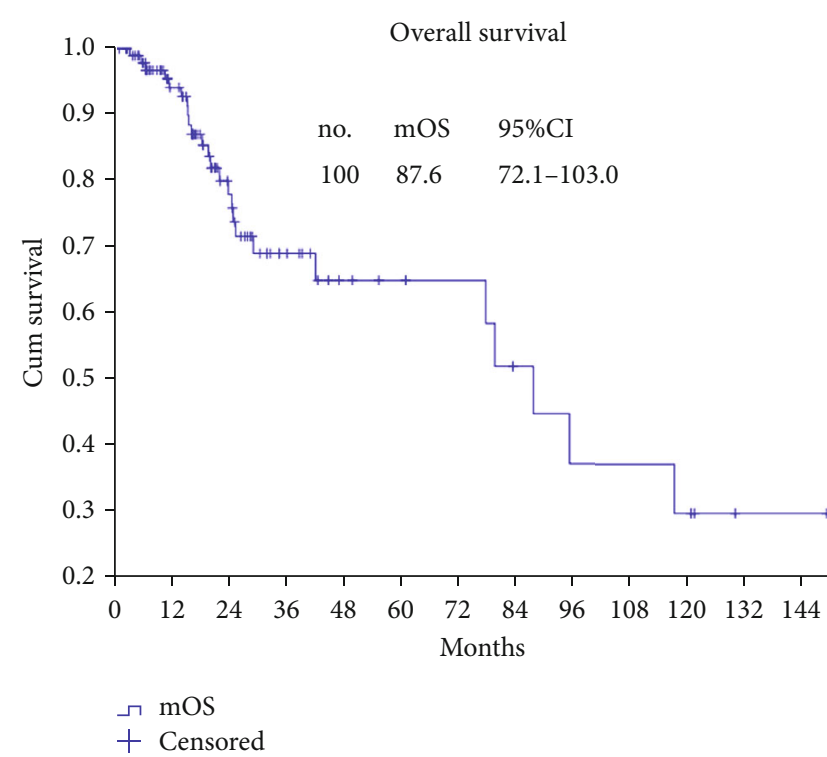

(a)

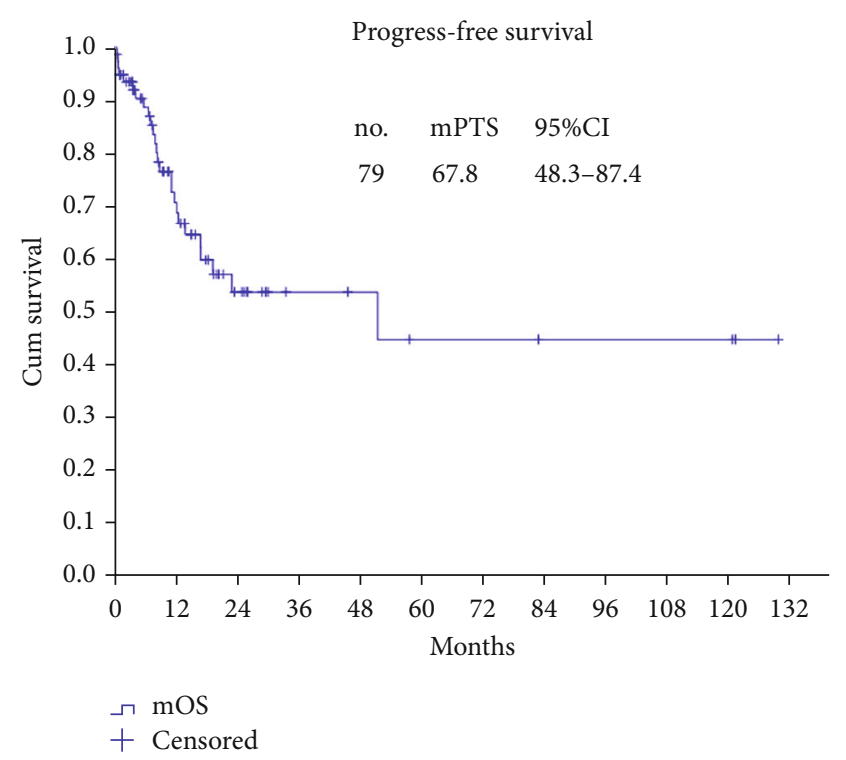

(b)

FIGURE 2: OS and PFS in the AOC patients. (a) OS of 100 AOC patients. (b) PFS of 79 AOC patients with complete CRS+HIPEC.

2.6. Study Parameters. (1) Clinicopathological characteristics: age, history of adjuvant therapy, KPS score, and preoperative tumor markers; (2) CRS+HIPEC-related parameters: duration of surgery, number of organs and peritoneal resected, number of anastomotic stoma, HIPEC regimens, PCI, CC score, and intraoperative volume; (3) survival: survival status, median overall survival ( $\mathrm{mOS}$ ), and median progression-free disease (mPFS); and (4) adverse events

2.7. Study Endpoints and Definition. (1) The primary endpoints of this study were OS and PFS. OS was defined as the time interval from the first surgery to tumor-related death or last follow-up. PFS was calculated from the date of surgery until the last follow-up that met the following criteria: the patients who underwent surgery-based curative comprehensive treatment developed any clinical manifestations, the CA 125 level rose again after surgery, medical imaging discovered any mass in the operation field, and the biopsy confirmed the diagnosis. (2) The secondary endpoints were perioperative serious adverse events (SAEs), which were defined as complications directly attributable to the treatment within 30 days of CRS+HIPEC and were evaluated based on the National Cancer Institute Common Terminology Criteria for Adverse Events version 4.0 [11]. (3) The PCI, according to Sugarbaker's criteria [8], is a standardized intraoperative staging system to determine the PM burden. The abdomen was divided into 13 areas, which included 9 sections of the abdominal cavity and 4 sections of the upper ileum, lower ileum, upper jejunum, and lower jejunum. The size of intraperitoneal nodules in each area was quantified. A score of 0 indicates that no malignant deposits are visualized; a score of 1 signifies that tumor nodules $\leq 0.5 \mathrm{~cm}$ are present; a score of 2 indicates that tumor nodules between 0.5 and $5.0 \mathrm{~cm}$ are present; a score of 3 signifies that tumor nodules $>5.0 \mathrm{~cm}$ in any dimension are present, and a confluence or layering of the tumor is scored as 3 . The maximum score is 39. (4) The CC score was defined as follows: a CC score of 0 indicates no visible residual peritoneal disease after CRS, a CC score of 1 indicates less than $2.5 \mathrm{~mm}$ of residual disease, a CC score of 2 indicates a residual tumor between $2.5 \mathrm{~mm}$ and $2.5 \mathrm{~cm}$, and a CC score of 3 indicates more than $2.5 \mathrm{~cm}$ of residual tumor or the presence of a sheet of unresectable tumor nodules [8] (Figure 1)

\section{Statistics Analysis}

The patient information was systematically integrated into a prospectively established database. Data analysis was conducted using the Statistical Package for Social Sciences version 24.0 (SPSS, Inc., Chicago, IL). Descriptive data are expressed as medians [range or 95\% confidence intervals (CIs)] for quantitative variables and as numbers (percentage) for qualitative data. The hypothesis test was performed by the $\chi^{2}$ test or Fisher's exact test. The Kaplan-Meier method was used to compare median survival with the log-rank test, and multivariate Cox regression analysis was performed to determine the independent predictors. The factors with $P<$ 0.05 in the univariate analysis were included in the multivariate analysis model. A two-sided $P<0.05$ was considered statistically significant.

\section{Results}

4.1. Characteristics of the AOC Patients. In total, $100 \mathrm{AOC}$ patients were treated with 106 CRS+HIPEC procedures, including 6 patients who each underwent 2 CRS+HIPEC procedures due to tumor recurrence. The median age was $58.5(28-87)$ years, and the median KPS score was 80 (50100); according to histopathological classification, 91 $(91 / 100,91.0 \%)$ patients had serous adenocarcinoma, and 9 $(9 / 100,9.0 \%)$ patients had other types of tumors. For the areas of surgical excision, the median number of organ 

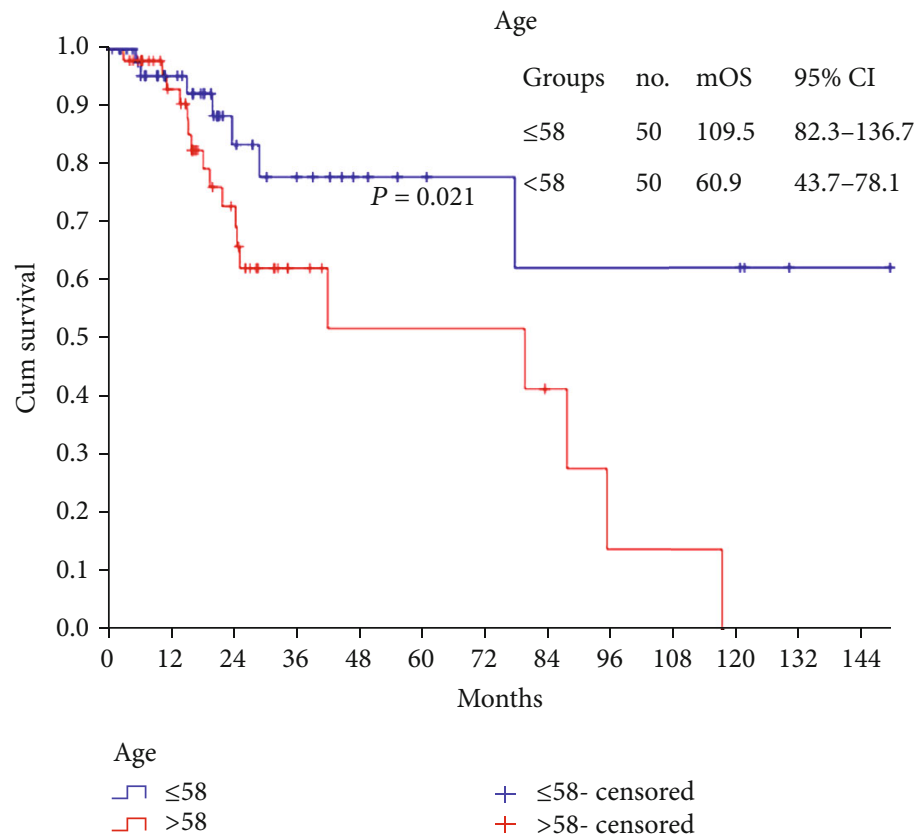

(a)

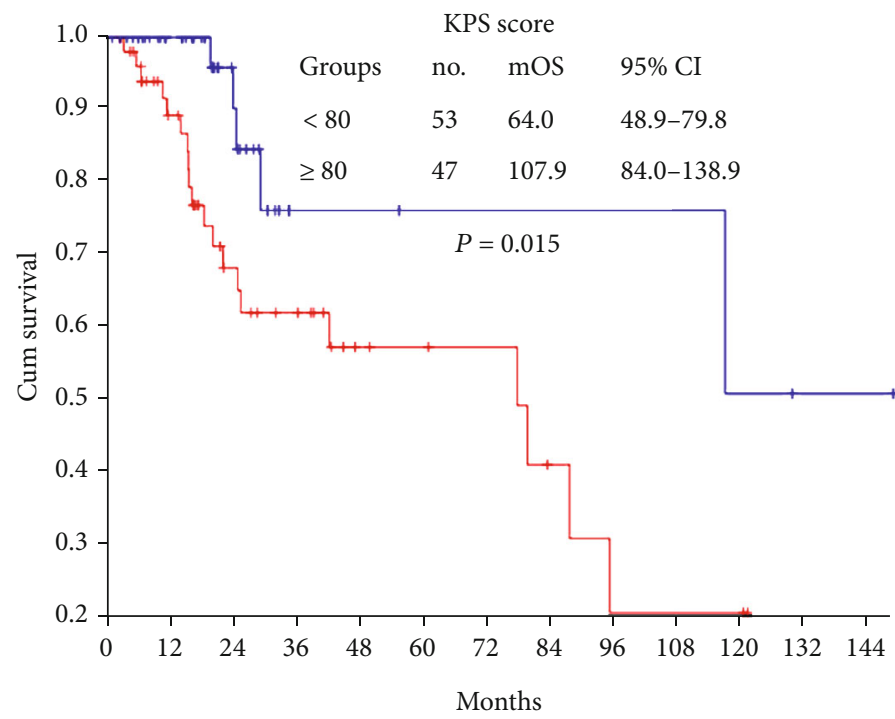

KPS score

$\neg \leq 80$

$+\leq 80$ - censored

$\neg>80$

$+>80$ - censored

(b)

Figure 3: Continued. 


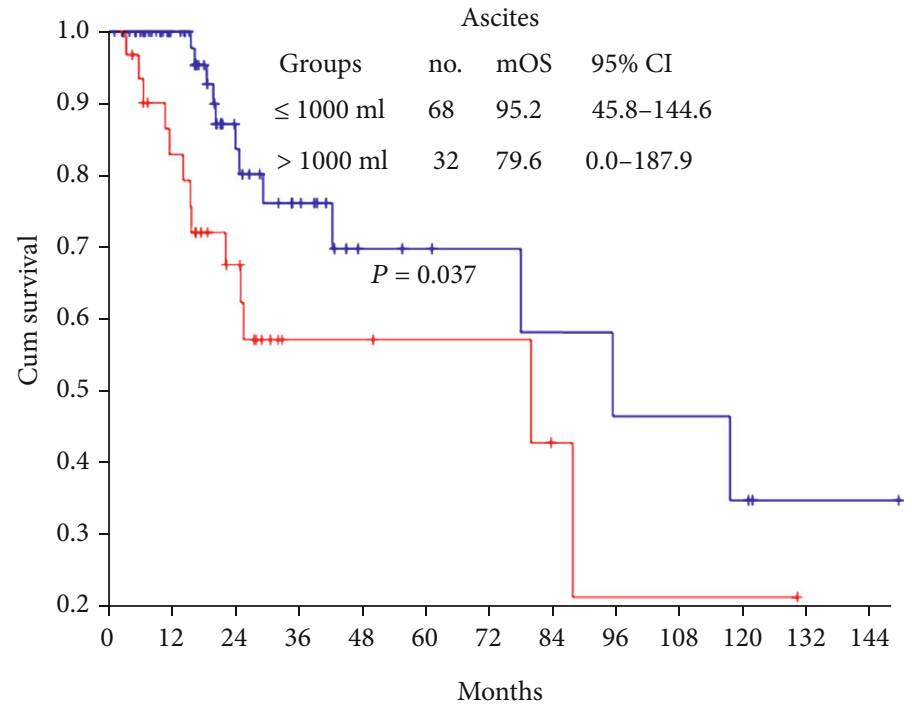

KPS score

$\neg \leq 1000 \mathrm{ml}$ $\neg>1000 \mathrm{ml}$

$+\leq 1000 \mathrm{ml}$ - censored

$+>1000 \mathrm{ml}$ - censored

(c)

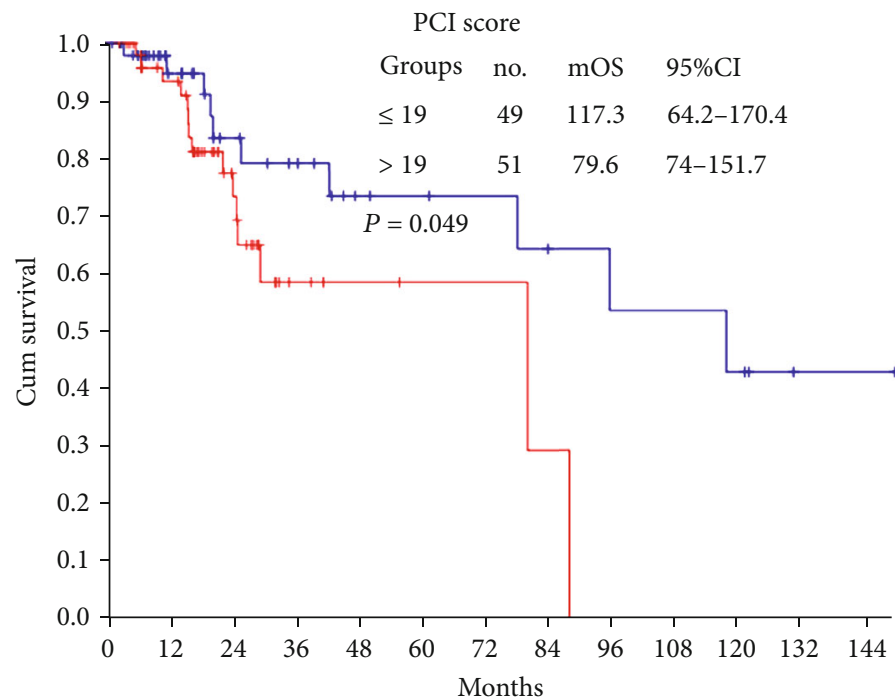

PCI score

$\neg \leq 19+\leq 19$ - censored

$+>19$ - censored

(d)

Figure 3: Continued. 

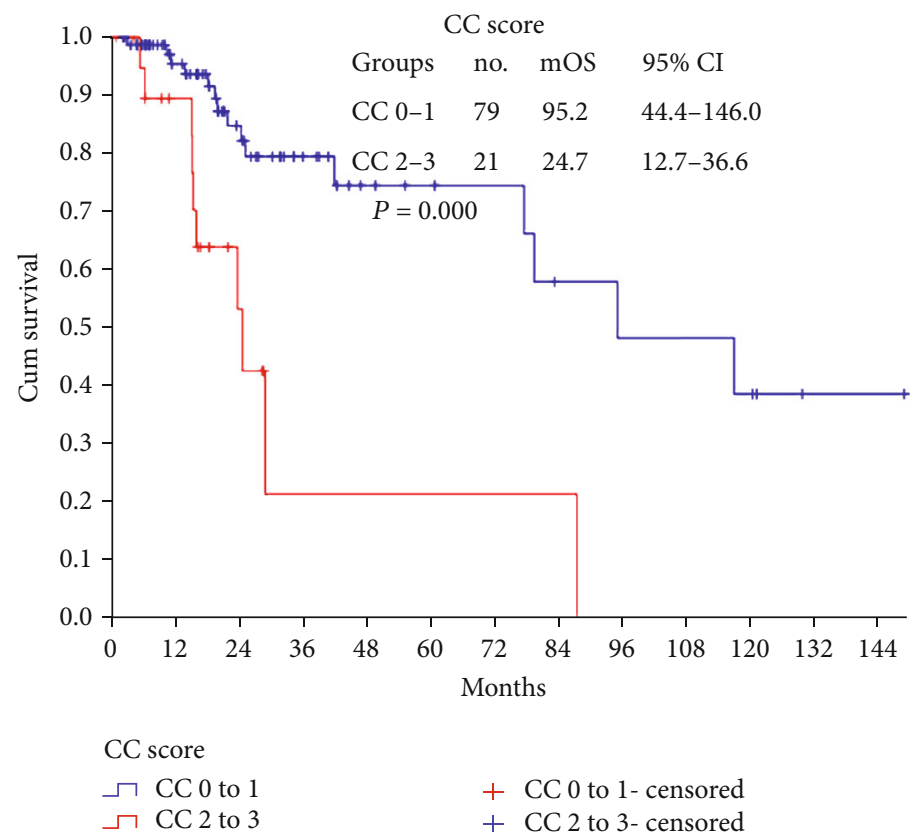

(e)
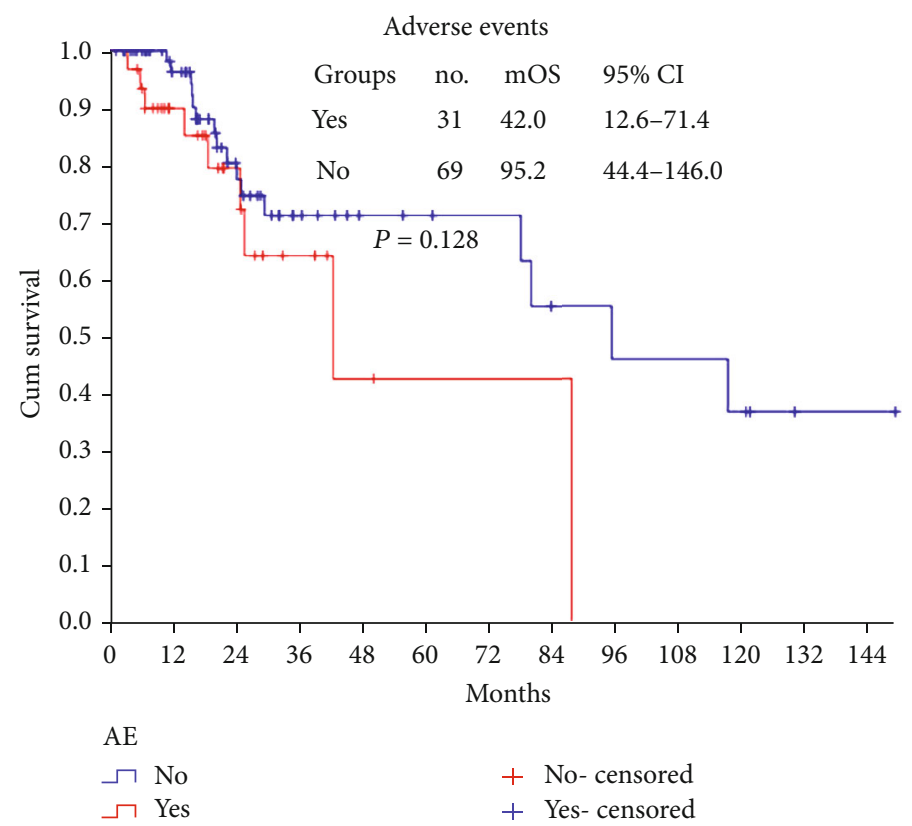

(f)

FIgUre 3: Overall survival of 100 AOC patients with correlation factors. (a) Age; (b) KPS score; (c) ascites; (d) PCI score; (e) CC score; (f) adverse events.

TABLE 2: Univariate and multivariate analyses on predictors of OS for 100 AOC patients.

\begin{tabular}{|c|c|c|c|c|c|c|c|c|}
\hline \multirow{2}{*}{ Variables } & \multicolumn{4}{|c|}{ Univariate analysis } & \multicolumn{4}{|c|}{ Multivariate analysis } \\
\hline & $\chi^{2}$ & $P$ & $\mathrm{HR}$ & $95 \% \mathrm{CI}$ & $\chi^{2}$ & $P$ & $\mathrm{HR}$ & $95 \% \mathrm{CI}$ \\
\hline CC score (CC 2-3 vs. CC 0-1) & 11.4 & $<0.001$ & 4.2 & $1.8-9.8$ & 6.8 & 0.009 & 3.2 & $1.3-7.5$ \\
\hline Age $(>58$ y $v s . \leq 58 \mathrm{y})$ & 4.8 & 0.021 & 2.7 & $1.1-6.5$ & & & & \\
\hline KPS score $(\geq 80 v s .<80)$ & 5.0 & 0.015 & 0.3 & $0.1-0.9$ & & & & \\
\hline PCI score $(>19 v s . \leq 19)$ & 3.8 & 0.049 & 2.5 & $1.0-6.0$ & & & & \\
\hline Ascites $(>1000 \mathrm{ml} v s . \leq 1000 \mathrm{ml})$ & 4.2 & 0.037 & 2.3 & $1.0-5.0$ & & & & \\
\hline
\end{tabular}

CI: confidence interval; HR: hazard ratio. 


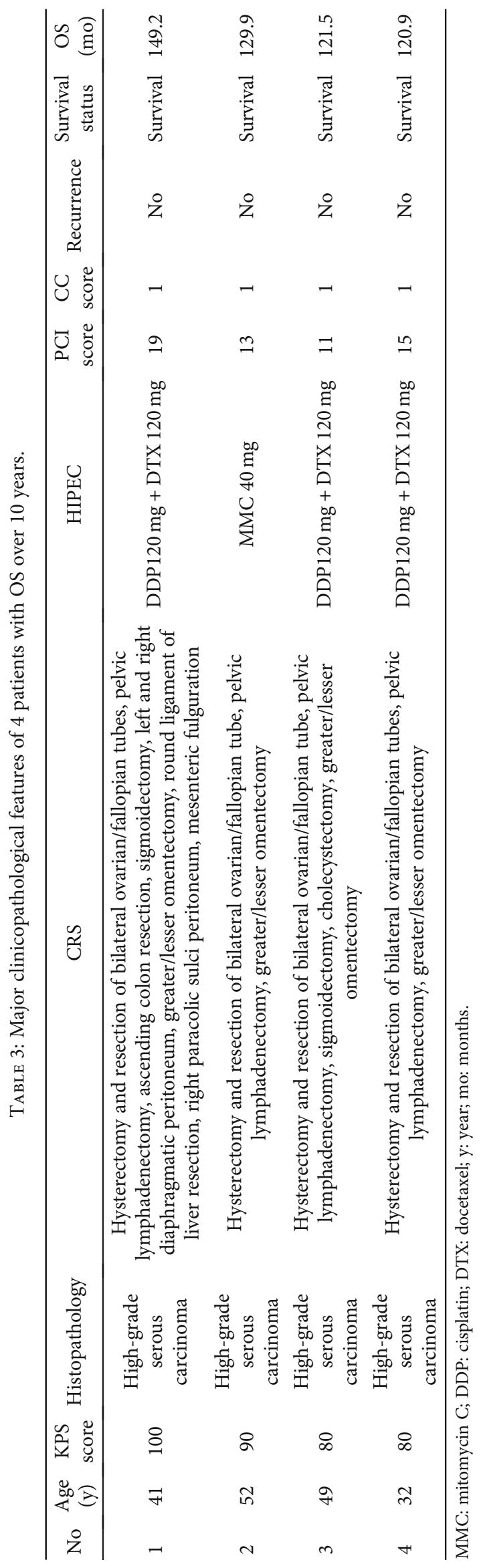


resections was 4 (3-9), and the number of peritoneal resections was 4 (1-9). Regarding adjuvant therapies, preoperatively, SC was applied in $48(48 / 100,48.0 \%)$ cases, and IPC was applied in $16(16 / 100,16.0 \%)$ cases; postoperatively, SC was applied in $76(76 / 100,76.0 \%)$ cases, and IPC was applied in $46.0(46 / 100,46.0 \%)$ cases. The clinicopathological characteristics are listed in Table 1.

4.2. Efficacy. The median follow-up was 36.8 (0.8-159.3) months. At the time of analysis, 25 (25/100, 25\%) patients had died, and $75(75 / 100,75.0 \%)$ patients were alive; the mOS was 87.6 (95\% CI: 72.1-103.0) months, and the 1-, 2-, $3-, 4-$, and 5- year survival rates were 94.1\%, 77.2\%, 68.2\%, $64.2 \%$, and $64.2 \%$, respectively (Figure 2 ).

4.3. Univariate and Multivariate Analyses for Predictors of OS. A univariate analysis identified 5 covariates indicative of improved survival, including age $\leq 58$ years (Figure 3(a), $P=0.021$ ), KPS score $\geq 80$ (Figure 3(b), $P=$ 0.015 ), ascites $\leq 1000 \mathrm{ml}$ (Figure $3(\mathrm{c}), P=0.037$ ), $P C I \leq 19$ (Figure 3(d), $P=0.049$ ), and CC score 0-1 (Figure 3(e), $P<0.001$ ) (Table 2). Multivariate Cox regression analysis identified the CC score as the only independent predictor for better survival. Compared with a CC score of 2-3, a CC score of $0-1$ was approximately 3.2 times $(P=0.009$, $\mathrm{HR}=3.2,95 \%$, and $\mathrm{CI}: 1.3-7.5)$ more likely to indicate improved survival (Table 2).

4.4. Special Analysis of Four Patients with an OS of over 10 Years. At the time of analysis, there were 4 (4/100, 4.0\%) patients with an OS of over 10 years and without any evidence of tumor recurrence; their OS durations were 149.2, $129.9,121.5$, and 120.9 months. The detailed clinical course of these four patients is listed in Table 3 .

4.5. Adverse Events. Adverse events (AEs) from grades I to V occurred in $31(31 / 100,31.0 \%)$ patients, including anemia and hypoproteinemia in $5(5 / 100,5.0 \%)$ patients, urinary fistula in $4(4 / 100,4.0 \%)$ patients, ileus in $4(4 / 100,4.0 \%)$ patients, respiratory infection in $4(4 / 100,4.0 \%)$ patients, deep vein thrombosis (DVT) in $3(3 / 100,3.0 \%)$ patients, wound infection in $3(3 / 100,3.0 \%)$ patients, renal dysfunction in $2(2 / 100,2.0 \%)$ patients, and urinary tract infection in $2(2 / 100,2.0 \%)$ patients. Grades III to V SAEs occurred in $4(4 / 100,4.0 \%)$ patients, including $2(2 / 100,2.0 \%)$ patients who died within 30 days of acute renal failure, $1(1 / 100,1.0 \%)$ patient with blood loss, and $1(1 / 100,1.0 \%)$ patient with ascending colon leakage. The detailed information is listed in Table 4.

4.6. Survival Analysis of Patients with CC Scores of 0-1. A subgroup analysis was conducted. Seventy-nine (79/100, 79.0\%) AOC patients achieved CC scores of $0-1$, the mPFS was 67.8 (95\% CI: 48.3-87.4) months (Figure 2(b)), and the mOS was 95.2 (95\% CI: 44.4-146.0) months (Figure 3(e)).

\section{Discussion}

The treatment of AOC remains an open and critical question. Despite clinical remission after palliative surgery and plati-
TABLE 4: Adverse events rate of 100 AOC patients.

\begin{tabular}{lc}
\hline Items & $n, \%$ \\
\hline SAE (grades III-V) & $4(4.0)$ \\
Perioperative mortality & $2(2.0)$ \\
Blood loss & $1(1.0)$ \\
Colon leakage & $1(1.0)$ \\
AE (grades I-II) & $27(27.0)$ \\
Anemia and hypoproteinemia & $5(5.0)$ \\
Urinary fistula & $4(4.0)$ \\
Ileus & $4(4.0)$ \\
Respiratory infection & $4(4.0)$ \\
DVT & $3(3.0)$ \\
Wound infection & $3(3.0)$ \\
Renal dysfunction & $2(2.0)$ \\
Urinary tract infection & $2(2.0)$ \\
\hline
\end{tabular}

AE: adverse event; SAE: serious adverse event; DVT: deep venous thrombosis.

num/taxane-based systematic chemotherapy $[5,13,14]$, the overall survival of patients with PM is very limited. At present, PM is no longer regarded as a form of systemic and widespread metastasis but a locoregional spread of abdominopelvic malignancies [15]. Accordingly, an integrated treatment strategy of CRS+HIPEC has been developed by pioneering oncologists and has become a standard treatment for malignant mesothelioma of the peritoneum and pseudomyxoma peritoneum and selected patients with colorectal cancer [16-19]. Gradually, the efficacy of CRS+HIPEC has been supported and promoted by various cancer centers for patients with AOC [20-22], but valuable new information and evidence, which could help in the process of selecting patients, formulating HIPEC regimens, normalizing standard surgical procedures, and evaluating safety, are urgently needed.

We aimed to investigate the efficacy and safety of CRS+HIPEC as a first-line surgery strategy in 100 patients with AOC. In essence, by comparing long-term survival, we were able to evaluate whether CRS+HIPEC, as a comprehensive therapy strategy, can be a suitable for the routine treatment for AOC. The results showed that the mOS was 87.6 months, and the 1-, 3-, and 5-year survival rates were $94.1 \%, 68.2 \%$, and $64.2 \%$, respectively. Complete CRS was achieved in $79.0 \%$ of patients, and the mPFS was 67.8 months. Of special note are four patients with high-grade serous disease who achieved an OS $>10$ years and were disease-free at the time of the most recent follow-up. The multimodality approach following traditional therapy achieved a mOS of 50 months and a mPFS of 3 to 4 months, 5 -year survival rates of over $30 \%$, and a relapse rate of $75 \%$. Our results indicated that CRS+HIPEC significantly prolongs the survival of patients with AOC compared to traditional treatment.

van Driel et al. [10] reported the first large-sample randomized controlled trial (RCT) on CRS+HIPEC in primary stage III OC in 2018. A total of 245 patients with newly diagnosed AOC (stage III), fallopian tube carcinoma, and 
TABLE 5: Previously published studies for AOC patients with complete CRS in recent 5 years.

\begin{tabular}{|c|c|c|c|c|c|c|c|}
\hline No. & Author & Year & No. (\%) & mOS (mo) & mPFS (mo) & SAE (\%) & Mortality (\%) \\
\hline 1 & Coccolini et al. [31] & 2015 & $54(100.0)$ & 32.9 & 12.5 & 35.2 & 5.6 \\
\hline \multirow[t]{2}{*}{2} & Kocic et al. [32] & 2016 & $28(96.8)$ & 51.0 & 19.0 & NA & NA \\
\hline & Sun et al. [33] & 2016 & $28(60.9)$ & 79.5 & 8.5 & 10.0 & 0.0 \\
\hline 3 & Manzanedo et al. [34] & 2017 & $59(97.0)$ & NA & 17.0 & NA & NA \\
\hline 4 & Magge et al. [35] & 2017 & $68(90.6)$ & 41.8 & 13.3 & NA & NA \\
\hline 5 & Pavlov et al. [2] & 2017 & $112(97.0)$ & 40.3 & 26.7 & 9.5 & 0.8 \\
\hline 6 & Di Giorgio et al. [36] & 2017 & $371(72.6)$ & 52.4 & 16.6 & 17.4 & 0.0 \\
\hline 7 & Mendivil et al. [24] & 2017 & $68(100.0)$ & 33.8 & 25.1 & 0.0 & 0.0 \\
\hline 8 & Mercier et al. [16] & 2018 & $155(92.5)$ & 69.3 & 30.3 & NA & NA \\
\hline 9 & van Driel et al. [10] & 2018 & $106(87.0)$ & 45.7 & 14.2 & 27.0 & 0.0 \\
\hline 10 & This study & 2019 & $79(79.0)$ & 95.2 & 67.8 & 4.0 & 2.0 \\
\hline
\end{tabular}

NA: not available; mo: months.

primary peritoneal carcinoma were treated with CRS+HIPEC (DDP $100 \mathrm{mg}, 40^{\circ} \mathrm{C}$, and $90 \mathrm{~min}$ ) following neoadjuvant chemotherapy. The mOS was 45.7 months for 106 (87.0\%) patients with CC scores of $0-1$, and the SAE rate was $27.0 \%$. In our study, a mOS of 87.6 months and a mPFS of 67.8 months were achieved, and these results were better than those in previous studies. Two points deserve special attention. First, our patients underwent radical resection with strict surgical procedures to strive for complete CRS. This leaves a minimal residual tumor burden after surgery. Second, complete and nearly complete CRS was immediately followed by HIPEC with drug combinations at a temperature of $43^{\circ} \mathrm{C}$. The synergistic effects of DDP, DTX, hyperthermia, and radical resection could produce significantly better survival benefits not comparable to those of any other treatment modalities applied individually.

The more extensive surgery to minimize tumor burden led to the success of CRS+HIPEC, which is an independent prognostic factor for patients with AOC [23]. In recent years, CRS+HIPEC has also been evaluated in the settings of primary AOC in several studies with variable results, showing that the mOS after complete CRS that achieves a CC score of $0-1$ was 32.9 to 79.5 months, and the 5 -year survival rate was 12.0 to $66.0 \%[9,23-27]$ (Table 5). Our study also showed favorable survival for patients with CC scores $0-1$ who had a mOS of 95.2 months, and the 1-, 3-, and 5-year survival rates were $95.4 \%, 78.7 \%$, and $73.8 \%$, respectively. Additionally, the multivariate Cox regression analysis identified the CC score as an independent factor for better survival, which is in accordance with the literature reports. In addition, compared with a CC score of 2-3, a CC score $0-1$ was approximately 3.2 times more likely to indicate improved survival. All of these results indicated that complete CRS was key to better survival.

As such, every attempt should be made to achieve complete CRS, which means a high risk for AEs and mortality. The safety of CRS+HIPEC has been fully verified, with a perioperative mortality rate of 0 to $10.0 \%$ and an incidence of SAEs of $22.0 \%$ to $28.0 \%$ [18, 34-37]. In our experience, the incidence of grades I to V AEs was $31.0 \%$, and $4.0 \%$ of patients developed SAEs, with a perioperative mortality rate of $2.0 \%$, which is similar to that in literature reports. Although we observed no significant differences in patient survival, the mOS of patients without AEs was 53.2 months longer than that of patients with AEs (Figure 3(f)). Hence, further studies are necessary to confirm that AEs may have a detrimental impact on survival.

Apart from its retrospective design, the limitations of this study are the use of single-center cohort with a short median follow-up, but the results showed a tendency towards long survival and safety benefits for AOC patients who underwent CRS+HIPEC as an upfront surgery strategy at experienced high-volume peritoneal cancer centers.

\section{Conclusions}

In summary, this study has provided evidence that CRS+HIPEC, as a preferred surgical strategy, could prolong the survival of AOC patients, especially for those with a KPS score $>80$, low PCI, and complete CRS. Therefore, strict patient selection and complete CRS in specialized peritoneal cancer centers are key factors for better survival.
Abbreviations
OC: $\quad$ Ovarian cancer
PC: $\quad$ Peritoneal carcinoma
AOC: Advanced ovarian cancer
CRS: Cytoreductive surgery
HIPEC: Hyperthermic intraperitoneal chemotherapy
ULN: Upper limit of normal
ALT: Alanine aminotransferase
AST: Aspartic aminotransferase
Scr: $\quad$ Serum creatinine
KPS: Karnofsky performance status
CA 125: Carbohydrate antigen 125
CA 199: Carbohydrate antigen 199
CEA: Carcinoembryonic antigen
CT: Computed tomography
DTX: Docetaxel
MMC: Mitomycin C
DDP: Cisplatin 


$\begin{array}{ll}\text { SC: } & \text { Systematic chemotherapy } \\ \text { IPC: } & \text { Intraperitoneal chemotherapy } \\ \text { OS: } & \text { Overall survival } \\ \text { PFS: } & \text { Progress-free survival } \\ \text { AEs: } & \text { Adverse events } \\ \text { SAEs: } & \text { Serious adverse events } \\ \text { PCI: } & \text { Peritoneal cancer index } \\ \text { CC: } & \text { Completeness of cytoreduction } \\ \text { CIs: } & \text { Confidence intervals. }\end{array}$

\section{Data Availability}

The datasets used and analyzed during the current study are available from the corresponding author on reasonable request.

\section{Ethical Approval}

The protocol of this prospective investigation was granted by the Institutional Review Board at Beijing Shijitan Hospital (code number 2019054). All methods were performed in accordance with the relevant guidelines and regulations or Declaration of Helsinki as patients were involved.

\section{Consent}

Written informed consent was obtained from all subjects or, if subjects are under 18, from a parent and/or legal guardian.

\section{Disclosure}

This manuscript has been presented as a preprint in http://Researchsquare.com.

\section{Conflicts of Interest}

The authors declare that they have no competing interests.

\section{Acknowledgments}

This study was supported by the grants supporting Beijing Municipal Administration of Hospitals' Ascent Plan (DFL20180701), Special Fund for the Capital Characteristic Clinical Medicine Development Project (Z161100000516077), Beijing Municipal Grant for Medical Talents Group on Peritoneal Surface Oncology (2017400003235J007), Key Discipline Development Fund of Beijing Shijitan Hospital affiliated to the Capital Medical University (2016fmzlwk), Beijing Natural Science Foundation (7172108), Health Science Promotion Project of Beijing (2018-TG-27), and Hubei Provincial Natural Science Foundation of China (2017CFB177). Thanks for the support of journal of Cancer Research and Clinic (a domestic journal of China), in which the data from this article was published on November, 2020, issue 32 (8): 574-578. With the permission and authorization of the journal, we updated the data and translated it into English and submitted to the journal of Biomed Research International.

\section{References}

[1] L. A. Torre, B. Trabert, C. E. DeSantis et al., "Ovarian cancer statistics, 2018," CA: a Cancer Journal for Clinicians, vol. 68, no. 4, pp. 284-296, 2018.

[2] M. J. Pavlov, M. S. Ceranic, S. M. Latincic, P. V. Sabljak, D. M. Kecmanovic, and P. H. Sugarbaker, "Cytoreductive surgery and hyperthermic intraperitoneal chemotherapy for the treatment of advanced epithelial and recurrent ovarian carcinoma: a single center experience," International Journal of Hyperthermia, vol. 34, no. 5, pp. 564-569, 2018.

[3] W. F. Morano, M. Khalili, D. S. Chi, W. B. Bowne, and J. Esquivel, "Clinical studies in CRS and HIPEC: trials, tribulations, and future directions- a systematic review," Journal of Surgical Oncology, vol. 117, no. 2, pp. 245-259, 2018.

[4] J. GOH, G. R. MOHAN, R. LADWA, S. ANANDA, P. A. COHEN, and S. BARON-HAY, "Frontline treatment of epithelial ovarian cancer," Asia-Pacific Journal of Clinical Oncology, vol. 11, Suppl 6, pp. 1-16, 2015.

[5] D. M. Provencher, C. J. Gallagher, W. R. Parulekar et al., "OV21/PETROC: a randomized gynecologic cancer intergroup phase II study of intraperitoneal versus intravenous chemotherapy following neoadjuvant chemotherapy and optimal debulking surgery in epithelial ovarian cancer," Annals of Oncology, vol. 29, no. 2, pp. 431-438, 2018.

[6] P. Cascales-Campos, V. López-López, J. Gil et al., "Hyperthermic intraperitoneal chemotherapy with paclitaxel or cisplatin in patients with stage III-C/IV ovarian cancer. Is there any difference?," Surgical Oncology, vol. 25, no. 3, pp. 164-170, 2016.

[7] G. S. Kireeva, G. I. Gafton, K. D. Guseynov et al., "HIPEC in patients with primary advanced ovarian cancer: is there a role? A systematic review of short- and long-term outcomes," Surgical Oncology, vol. 27, no. 2, pp. 251-258, 2018.

[8] P. H. Sugarbaker, "Cytoreductive surgery and perioperative intraperitoneal chemotherapy for the treatment of advanced primary and recurrent ovarian cancer," Current Opinion in Obstetrics \& Gynecology, vol. 21, no. 1, pp. 15-24, 2009.

[9] N. Bakrin, J. M. Bereder, E. Decullier et al., "Peritoneal carcinomatosis treated with cytoreductive surgery and hyperthermic intraperitoneal chemotherapy (HIPEC) for advanced ovarian carcinoma: a French multicentre retrospective cohort study of 566 patients," European Journal of Surgical Oncology, vol. 39, no. 12, pp. 1435-1443, 2013.

[10] W. J. van Driel, S. N. Koole, K. Sikorska et al., "Hyperthermic intraperitoneal chemotherapy in ovarian cancer," The New England Journal of Medicine, vol. 378, no. 3, pp. 230-240, 2018.

[11] NCCN guidelines, Ovarian cancer, NCCN, America, 2019, org/patients/survey/.

[12] Y. Li, Y. F. Zhou, H. Liang et al., "Chinese expert consensus on cytoreductive surgery and hyperthermic intraperitoneal chemotherapy for peritoneal malignancies," World Journal of Gastroenterology, vol. 22, no. 30, pp. 6906-6916, 2016.

[13] H. Yao and J. Ma, "Dendrimer-paclitaxel complexes for efficient treatment in ovarian cancer: study on OVCAR-3 and HEK293T cells," Acta Biochimica Polonica, vol. 65, no. 2, pp. 219-225, 2019.

[14] M. Petrillo, L. P. Anchora, G. Scambia, and A. Fagotti, "Cytoreductive surgery plus platinum-based hyperthermic intraperitoneal chemotherapy in epithelial ovarian cancer: a promising integrated approach to improve locoregional control," The Oncologist, vol. 21, no. 5, pp. 532-534, 2016. 
[15] P. H. Sugarbaker, "Peritoneal metastases from gastrointestinal cancer," Current Oncology Reports, vol. 20, no. 8, p. 62, 2018.

[16] the PSOGI Working Group, the BIG-RENAPE Working Group, F. Mercier, N. Bakrin et al., "Peritoneal carcinomatosis of rare ovarian origin treated by cytoreductive surgery and hyperthermic intraperitoneal chemotherapy: a multiinstitutional cohort from PSOGI and BIG-RENAPE," Annals of Surgical Oncology, vol. 25, no. 6, pp. 1668-1675, 2018.

[17] on behalf of the RENAPE Network, B. Malgras, E. Gayat et al., "Impact of combination chemotherapy in peritoneal mesothelioma hyperthermic intraperitoneal chemotherapy (HIPEC): the RENAPE study," Annals of Surgical Oncology, vol. 25, no. 11, pp. 3271-3279, 2018.

[18] C. Q. Huang, Y. Min, S. Y. Wang et al., "Cytoreductive surgery plus hyperthermic intraperitoneal chemotherapy improves survival for peritoneal carcinomatosis from colorectal cancer: a systematic review and meta-analysis of current evidence," Oncotarget, vol. 8, no. 33, pp. 55657-55683, 2017.

[19] Y. Wang, F. Ren, P. Chen, S. Liu, Z. Song, and X. Ma, "Effects of cytoreductive surgery plus hyperthermic intraperitoneal chemotherapy (HIPEC) versus cytoreductive surgery for ovarian cancer patients: a systematic review and meta-analysis," European Journal of Surgical Oncology, vol. 45, no. 3, pp. 301-309, 2019.

[20] M. Deraco, S. Sinukumar, R. A. Salcedo-Hernández et al., "Clinico-pathological outcomes after total parietal peritonectomy, cytoreductive surgery and hyperthermic intraperitoneal chemotherapy in advanced serous papillary peritoneal carcinoma submitted to neoadjuvant systemic chemotherapy- largest single institute experience," European Journal of Surgical Oncology, vol. 45, no. 11, pp. 2103-2108, 2019.

[21] R. Pozzar, L. M. Baldwin, B. A. Goff, and D. L. Berry, "Patient, physician, and caregiver perspectives on ovarian cancer treatment decision making: lessons from a qualitative pilot study," Pilot Feasibility Stud., vol. 4, no. 1, p. 91, 2018.

[22] K. Jaaback, N. Johnson, and T. A. Lawrie, "Intraperitoneal chemotherapy for the initial management of primary epithelial ovarian cancer," Cochrane Database of Systematic Reviews, vol. 1, article CD005340, 2016.

[23] D. Biacchi, F. Accarpio, L. Ansaloni et al., "Upfront debulking surgery versus interval debulking surgery for advanced tuboovarian high-grade serous carcinoma and diffuse peritoneal metastases treated with peritonectomy procedures plus HIPEC," Journal of Surgical Oncology, vol. 120, no. 7, pp. 1208-1219, 2019.

[24] A. A. Mendivil, M. A. Rettenmaier, L. N. Abaid et al., "Consolidation hyperthermic intraperitoneal chemotherapy for the treatment of advanced stage ovarian carcinoma: a 3 year experience," Cancer Chemotherapy and Pharmacology, vol. 80, no. 2, pp. 405-410, 2017.

[25] R. A. Cowan, R. E. O'Cearbhaill, O. Zivanovic, and D. S. Chi, "Current status and future prospects of hyperthermic intraoperative intraperitoneal chemotherapy (HIPEC) clinical trials in ovarian cancer," International Journal of Hyperthermia, vol. 33, no. 5, pp. 548-553, 2017.

[26] M. Markman, "Hyperthermic intraperitoneal chemotherapy in ovarian cancer: where do we go from here?," The Oncologist, vol. 21, no. 5, pp. 529-531, 2016.

[27] A. Bhatt and O. Glehen, "The role of cytoreductive surgery and hyperthermic intraperitoneal chemotherapy (HIPEC) in ovarian cancer: a review," Indian Journal of Surgical Oncology, vol. 7, no. 2, pp. 188-197, 2016.
[28] M. Ba, H. Long, X. Zhang et al., "Hyperthermic intraperitoneal perfusion chemotherapy and cytoreductive surgery for controlling malignant ascites from ovarian cancer," International Journal of Gynecological Cancer, vol. 26, no. 9, pp. 15711579, 2016.

[29] P. A. Cascales-Campos, J. Gil, E. Gil et al., "Treatment of microscopic disease with hyperthermic intraoperative intraperitoneal chemotherapy after complete cytoreduction improves disease-free survival in patients with stage IIIC/IV ovarian cancer," Annals of Surgical Oncology, vol. 21, no. 7, pp. 2383-2389, 2014.

[30] P. Cascales Campos, J. Gil, and P. Parrilla, "Morbidity and mortality outcomes of cytoreductive surgery and hyperthermic intraperitoneal chemotherapy in patients with primary and recurrent advanced ovarian cancer," European Journal of Surgical Oncology, vol. 40, no. 8, pp. 970-975, 2014.

[31] F. Coccolini, L. Campanati, F. Catena et al., "Hyperthermic intraperitoneal chemotherapy with cisplatin and paclitaxel in advanced ovarian cancer: a multicenter prospective observational study," Journal of Gynecologic Oncology, vol. 26, no. 1, pp. 54-61, 2015.

[32] M. Kocic, S. Nikolic, M. Zegarac et al., "Prognostic factors and outcomes of cytoreductive surgery combined with hyperthermic intraperitoneal chemotherapy in patients with advanced ovarian cancer - a single tertiary institution experience," Journal of BUON, vol. 21, no. 5, pp. 1176-1183, 2016.

[33] J. H. Sun, Z. H. Ji, Y. Yu et al., "Cytoreductive surgery plus hyperthermic intraperitoneal chemotherapy to treat advance$\mathrm{d} /$ recurrent epithelial ovarian cancer: results from a retrospective study on prospectively established database," Translational Oncology, vol. 9, no. 2, pp. 130-138, 2016.

[34] I. Manzanedo, F. Pereira, E. Pérez-Viejo et al., "Hyperthermic intraoperative intraperitoneal chemotherapy (HIPEC) with primary or secondary cytoreductive surgery in the treatment of advanced epithelial ovarian cancer," Minerva Ginecologica, vol. 69, no. 2, pp. 119-127, 2017.

[35] D. Magge, L. Ramalingam, Y. Shuai et al., "Hyperthermic intraperitoneal chemoperfusion as a component of multimodality therapy for ovarian and primary peritoneal cancer," Journal of Surgical Oncology, vol. 116, no. 3, pp. 320-328, 2017.

[36] A. di Giorgio, P. de Iaco, M. de Simone et al., "Cytoreduction (peritonectomy procedures) combined with hyperthermic intraperitoneal chemotherapy (HIPEC) in advanced ovarian cancer: retrospective Italian multicenter observational study of 511 cases," Annals of Surgical Oncology, vol. 24, no. 4, pp. 914-922, 2017.

[37] National cancer institute, "Common terminology critertia for adverse events (CTCAE) v5.0," 2017, https://ctep.cancer.gov/ protocolDevelopment/electronic_applications/ctc.htm\#ctc_50. 